\title{
Patients experiencing early acute respiratory failure have high postoperative mortality after pneumonectomy
}

Kim Blanc, MD, ${ }^{\mathrm{a}}$ Agnès Dechartres, MD, PhD, ${ }^{\mathrm{b}}$ Rym Zaimi, MD, ${ }^{\mathrm{c}}$ Aurélie Lefebvre, MD, ${ }^{\mathrm{a}}$ Aurélie Janet-Vendroux, MD, ${ }^{\mathrm{c}}$ Ludovic Fournel, MD, ${ }^{\mathrm{c}}$ Hervé Dermine, MD, ${ }^{\mathrm{d}}$ Christine Lorut, MD, ${ }^{\mathrm{a}}$ Xavier Becanne, MD, ${ }^{\mathrm{d}}$ Emelyne Hamelin-Canny, $\mathrm{MD},{ }^{\mathrm{c}}$ Antonio Bobbio, MD, PhD, Nicolas Roche, MD, PhD, ${ }^{\mathrm{a}}$ Jean-François Regnard, $\mathrm{MD},{ }^{\mathrm{c}}$ Marco Alifano, $\mathrm{MD}, \mathrm{PhD},{ }^{\mathrm{c}}$ and Antoine Rabbat, MD

\section{ABSTRACT}

Objective: Post-pneumonectomy acute respiratory failure leading to invasive mechanical ventilation carries a severe prognosis especially when acute respiratory distress syndrome occurs. The aim of this study was to describe risk factors and outcome of acute respiratory failure.

Methods: We retrospectively reviewed clinical files of all patients who underwent pneumonectomy in a single center between 2005 and 2015. Risk factors and outcome of acute respiratory failure were assessed in univariate and multivariate analysis.

Results: Among the 543 patients who underwent pneumonectomy in the period of study, $89(16.4 \%)$ needed reintubation within the 30th postoperative day and 60 of these (11\% of all pneumonectomies) developed acute respiratory distress syndrome. In multivariate analysis, right-side of pneumonectomy (odds ratio [OR], 2.29; $95 \%$ confidence interval [CI], 1.24-4.22), chronic cardiac disease (OR, 2.15; 95\% CI, 1.08-4.25), Charlson Comorbidity Index (OR, 1.35; 95\% CI, 1.14-1.61), carinal resection (OR, 3.23; 95\% CI, 1.26-8.29), and extrapleural pneumonectomy (OR, 8.36; 95\% CI, 3.31-21.11) were identified as independent risk factors of reintubation. Thirty-day mortality was $7.7 \%$ for all pneumonectomies, $41.6 \%$ (37/89) in the invasive ventilation group, and 53.3\% (32/60) in patients with acute respiratory distress syndrome. In non-reintubated patients, 30 -day mortality was $1.1 \%(5 / 454)$. In reintubated patients, 5 -year survival was $27.1 \%$ (95\% CI, 17.8-41.4).

Conclusions: Early acute respiratory failure requiring reintubation remains a severe complication of pneumonectomy with a poor outcome. (J Thorac Cardiovasc Surg 2018;156:2368-76)

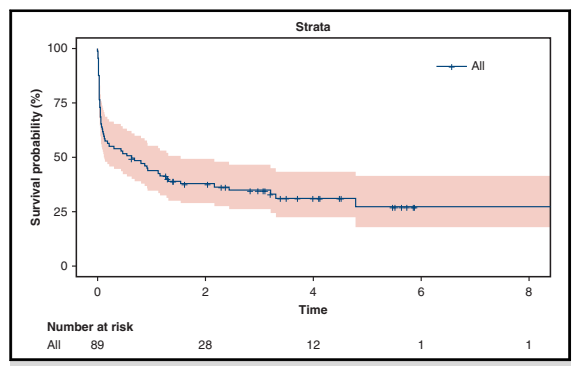

Overall survival Kaplan-Meier curve in pneumonectomy patients requiring intubation.

\section{Central Message}

Acute respiratory failure occurring after pneumonectomy is associated with a poor outcome. Right-side pneumonectomy, comorbidities, and extended resection are independent risk factors of reintubation.

\section{Perspective}

Acute respiratory failure leading to invasive ventilation occurs not infrequently after pneumonectomy and is associated with high postoperative mortality. Right side, chronic cardiac disease, Charlson comorbidity index, carinal resection, and extrapleural pneumonectomy are independent risk factors of reintubation. Identification of high-risk patients could help in selection of surgical candidates.

See Editorial Commentary page 2377.

See Editorial page 2366.
From the Departments of ${ }^{\mathrm{a}}$ Chest Disease and Respiratory Intensive Care, ${ }^{\mathrm{c}}$ Thoracic Surgery, and ${ }^{\mathrm{d}}$ Anesthesia and Surgical Intensive Care, Cochin Hospital, and ${ }^{\mathrm{b}} \mathrm{Clinic}$ Epidemiology Center, Hôtel Dieu, HUPC, APHP, Paris Descartes University, Paris, France.

Received for publication June 14, 2016; revisions received Aug 5, 2018; accepted for publication Aug 17, 2018.

Address for reprints: Marco Alifano, MD, PhD, Service de chirurgie thoracique, Hôpitaux Universitaires, Paris Centre Hôpital, Cochin 27 rue du Faubourg Saint Jacques, 75679 Paris cedex 14, France (E-mail: marco.alifano@cch.aphp.fr). $0022-5223 / \$ 36.00$

Copyright $₫ 2018$ Published by Elsevier Inc. on behalf of The American Association for Thoracic Surgery

https://doi.org/10.1016/j.jtcvs.2018.08.113
Lung resection is associated with high postoperative morbidity. Postoperative pulmonary complications such as

Scanning this $\mathrm{QR}$ code will take you to the article title page to access supplementary information.

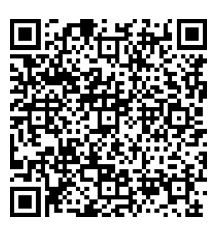




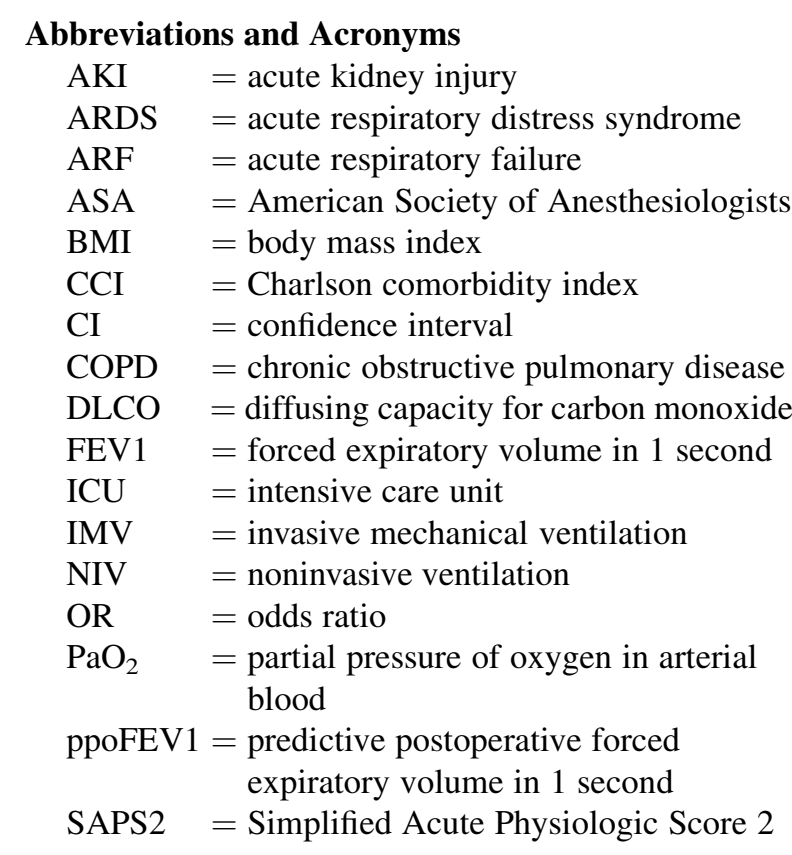

pneumonia or atelectasis are frequent and severe. ${ }^{1,2}$ Clinical studies have identified numerous patient- or procedurerelated risk factors of postoperative complications. ${ }^{1-7}$ After lung resection, acute respiratory failure (ARF) requiring invasive mechanical ventilation (IMV) carries a very high mortality rate (20\%-50\%), especially when acute respiratory distress syndrome (ARDS) occurs. ${ }^{8-11}$ IMV is clearly associated with many subsequent lifethreatening complications, such as infections, persistent air leaks, and bronchopleural fistula. ${ }^{1,12}$

Among pulmonary resections, pneumonectomy is associated with the highest postoperative mortality, with figures ranging from $6 \%$ to $9 \% .{ }^{13}$ With respect to long-term outcome, 5-year survival after pneumonectomy for lung cancer is approximately $35 \% .{ }^{13}$ Thus, it seems necessary to better understand factors associated with short-term outcome, to properly select patients for pneumonectomy.

Few studies have addressed risk factors for postpneumonectomy respiratory failure. ${ }^{10,11,14-17}$ Although the role of intraoperative nonprotective ventilation (large tidal volume and increased airway pressures) and fluid administration (nonrestriction) in the prevention of ARF and ARDS has been proven, ${ }^{17,18}$ patient-related preoperative factors remain a matter of debate, and even studies with many patients included often failed to find significant correlations. ${ }^{11}$ Furthermore, only a limited number of studies have focused on the outcome of patients with postpneumonectomy ARF. ${ }^{8,9,11,19}$ Most of them were performed several years ago and included a limited number of patients; furthermore, potentially important prognostic data were often missing. Last, during the past years, management of ARF has markedly changed. Thus, we aimed to assess: (1) risk factors of postoperative reintubation in pneumonectomy patients, mainly with a focus on preoperative functional assessment; and (2) short- and long-term outcomes of patients who required reintubation and IMV after pneumonectomy (Video 1).

\section{METHODS \\ Study Design and Patients}

This study was a single-center retrospective cohort study. Files from all consecutive adult patients who underwent pneumonectomy for cancer in our institution between January 2005 and December 2015 were retrospectively retrieved. There was no exclusion criterion. The research was conducted according to recommendations outlined in the Declaration of Helsinki as well as to French laws on Biomedical Research. Institutional review board approval was obtained (CERC-SFCTCV-2015-11-4-13-1616-16AlMa/CPP HUPC). All patients or relatives (in case of deceased patients) gave their informed consent to participate and allowed the use of their data.

\section{Preoperative Evaluation}

For every patient, preoperative evaluation consisted of: symptom-taking and physical examination, routine blood tests, pulmonary function assessment, and lung perfusion scan. Cardiac assessment was performed using clinical evaluation, electrocardiogram, and transthoracic echocardiography. A coronarography or cardiac stress test was performed when indicated. Patients were considered suitable for surgery if predicted postoperative forced expiratory volume in 1 second (FEV1) was $>40 \%$ of predicted, and there was neither hypercapnia $(>46 \mathrm{~mm} \mathrm{Hg}$ ) nor pulmonary hypertension. ${ }^{20}$ Patients were considered not suitable for surgery if predictive postoperative FEV1 (ppoFEV1) was $<30 \%$. In intermediate cases, surgical indication was established in a multidisciplinary setting by taking into account results of lung diffusing capacity for carbon monoxide (DLCO) measure and low technology (6-minute shuttle test or star-climbing test) or high technology (maximal oxygen uptake) ergometric tests. Patients could benefit from pulmonary rehabilitation to improve their pulmonary function before surgery. Indications to surgery were established according to French Thoracic and Cardiovascular Society guidelines. $^{20}$

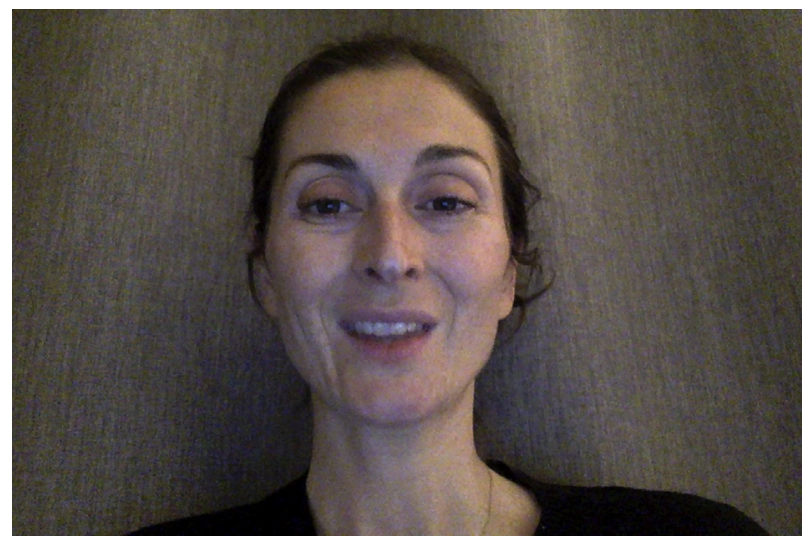

VIDEO 1. Why the article titled: "Patients experiencing early acute respiratory failure have high postoperative mortality after pneumonectomy" should be read. Video available at: https://www.jtcvs.org/article/S00225223(18)32508-X/fulltext. 


\section{Intraoperative Care}

All patients were intubated with a double-lumen tube. Perioperative antimicrobial prophylaxis was adminisered according to the local guidelines. When pleural space was opened, single-lung ventilation was used. Intraoperative ventilation was in positive-pressure mode. Plateau pressure was assimilated to peak pressure. Recruitment maneuvers could be performed when indicated. Fluid infusions were limited, and vasopressors were allowed. Surgical techniques were those usually used. A multimodal analgesia was administered including a thoracic paravertebral block at the end of surgery. Epidural analgesia was not systematic and was generally performed if a parietal resection was carried out, or if ppoFEV1 was between $30 \%$ and $40 \%$ of predicted. In almost all of the remaining patients, a paravertebral block was carried out. According to our fast-track procedure, most of the patients were extubated in the recovery room, or within a few hours after intensive care unit (ICU) admission.

\section{Postoperative Care}

All patients were hospitalized in our ICU after surgery. Postoperative management consisted of early mobilization, chest physiotherapy, aerosolized bronchodilators, and controlled multimodal analgesia including epidural or paravertebral block. If needed, supplemental oxygen was given through a nasal cannula to achieve oxygen saturation $>92 \%$. Oral realimentation was started on postoperative day 1 . Chest tube was withdrawn on postoperative day 2. Noninvasive ventilation (NIV) for ARF, before intubation, was introduced at the discretion of the clinicians.

\section{Data Collection and Outcome Measures}

We collected data on the following characteristics:

- Baseline patient characteristics: age, sex, smoking history including total pack-years, active smoking or past smoking status and date of withdrawal, body mass index (BMI; obesity was defined as BMI $>30$ ), chronic alcohol abuse (defined by a consumption $>30 \mathrm{~g} / \mathrm{d}$ for men, and $20 \mathrm{~g} / \mathrm{d}$ for women), diabetes mellitus, systemic arterial hypertension, chronic cardiac disease (defined as chronic atrial fibrillation or coronary artery disease), previous nonpulmonary cancer, Charlson comorbidity index (CCI), and American Society of Anesthesiologists (ASA) physical status.

- Respiratory function characteristics: presence of chronic obstructive pulmonary disease (COPD; diagnosis on the basis of a FEV1/forced vital capacity ratio $<70 \%$ ), preoperative FEV1, DLCO/alveolar volume (lung DLCO adjusted for alveolar volume, preoperative partial pressure of carbon dioxide in arterial blood value, ppoFEV1 calculated on the basis of preoperative FEV1 and results of lung perfusion scan).

- Tumor-related data: previous lung cancer, previous non-lung cancer, side of lung cancer, pathology: squamous cell carcinoma, adenocarcinoma, other histologic types of lung cancer, mesothelioma, metastasis, other malignancies; neoadjuvant chemotherapy or radiotherapy; pathologic stage.

- Types of resection: standard or extended pneumonectomy (combined resection of neighbouring organs, ie, pericardium, mediastinal fat, chest wall), extrapleural pneumonectomy, completion pneumonectomy, carinal resection.

- Data at ICU admission: patient characteristics at admission and occurrence of postoperative complications: pneumonia, atelectasis, COPD decompensation, cardiogenic pulmonary edema, pulmonary embolism, ARF, hemorrhagic shock, sepsis, acute kidney injury (AKI), acute coronary syndrome, and significant blood loss with need for transfusion. Finally we recorded indication for reintubation and IMV and NIV before orotracheal intubation (when performed).

- For reintubated patients, we also recorded at ICU admission: Simplified Acute Physiologic Score 2 (SAPS2) ${ }^{21}$ at admission, sepsis-related organ failure assessment score ${ }^{22}$ at day 1 and day 3 of admission. ARDS diagnosis and severity classification was on the basis of the Berlin ARDS definitions. $^{23}$
Thus, we identified patients who required early reintubation and IMV for postpneumonectomy ARF (within 30 days). Overall survival was assessed for each patient using direct phone interview or consultation of vital status registers of the municipality of birth. Survival was defined from the date of surgery and last alive contact or death.

\section{Statistical Analysis}

Descriptive analysis involved used of frequencies and percentages for categorical variables and mean $( \pm S D$ ) or median (quartile 1 to quartile 3 ) as appropriate for quantitative variables. We aimed to evaluate which preand intraoperative characteristics were independently associated with postoperative reintubation. To do so, we first analyzed relationships between need for reintubation and clinical and intraoperative characteristics of patients with $\chi^{2}$ tests or Fisher exact tests (as appropriate) for categorical variables, and Student $t$ test or Wilcoxon rank sum tests for quantitative variables. Selection of variables for the multivariate logistic regression model was on the basis of univariate analysis (inclusion of all variables associated with the event of interest with a $P$ value $<.05)$ and according to clinical judgement or literature review. Quantitative variables were kept in the models as quantitative variables. We evaluated colinearity by checking that the variance inflation factors were all $<2$ (indicating little to moderate correlation between variables). As the main multivariate analysis, we included all preselected variables but we also did a sensitivity analysis using a stepwise variable selection with likelihood ratio test $P$ value stopping rule of .1 to enter and .05 to remain in the final model. We evaluated the $c$ statistic as a measure of model performance. $P$ values were 2-tailed and $P<.05$ indicated statistical significance. Finally, we described survival in reintubated patients using a Kaplan-Meier curve from the date of surgery to the date of death or of last news. Statistical analysis involved use of SAS version 9.3 (SAS Institute Inc, Cary, NC) and $\mathrm{R}$ version 3.2.3 ( $\mathrm{R}$ Core Team (2015). R: A language and environment for statistical computing; $\mathrm{R}$ Foundation for Statistical Computing, Vienna, Austria; https://www.R-project.org).

\section{Definitions}

- ARF was defined by the presence and persistence of at least 2 of the following criteria: respiratory acidosis (arterial $\mathrm{pH}<7.35$ together with partial pressure of carbon dioxide in arterial blood $>50 \mathrm{~mm} \mathrm{Hg}$ ); arterial $\mathrm{O}_{2}$ saturation according to pulse oximetry of $<90 \%$ or partial pressure of oxygen in arterial blood $\left(\mathrm{PaO}_{2}\right)<60 \mathrm{~mm} \mathrm{Hg}$ at $\mathrm{FiO}_{2}$ of 0.5 or nasal oxygen therapy at $81 / \mathrm{min}$; respiratory rate $>30$ breaths per minute; clinical signs of ARF (ie, cyanosis, sweats, involvement of accessory respiratory muscles, paradoxical abdominal motion, consciousness impairment).

- ARDS was defined by the absence of hydrostatic cardiogenic pulmonary edema and $\mathrm{PaO}_{2} / \mathrm{FiO}_{2} \leq 300 \mathrm{~mm} \mathrm{Hg}$. ARDS was categorized in 3 groups according to Berlin classification: mild $\left(200 \mathrm{~mm} \mathrm{Hg}<\mathrm{PaO}_{2} / \mathrm{FiO}_{2}\right.$ $\leq 300 \mathrm{~mm} \mathrm{Hg})$ moderate $\left(100<\mathrm{PaO}_{2} / \mathrm{FiO}_{2} \leq 200 \mathrm{~mm} \mathrm{Hg}\right)$ and severe $\left(\mathrm{PaO}_{2} / \mathrm{FiO}_{2} \leq 100 \mathrm{~mm} \mathrm{Hg}\right)$. All $\mathrm{PaO}_{2} / \mathrm{FiO}_{2}$ were assessed with a positive expiratory pressure level $\geq 5 \mathrm{~cm} \mathrm{H}_{2} \mathrm{O}^{23}$

- Pneumonia was defined as the presence of new and/or progressive pulmonary infiltrates on chest radiography, plus 2 or more of the following criteria: fever $\left(>38^{\circ} \mathrm{C}\right)$, leukocytosis $\left(>12 \times 10^{9} / \mathrm{L}\right)$, purulent sputum, or isolation of pathogen in respiratory secretions.

- Atelectasis was defined as a reduction in lung volume, loss of aeration, and displacement in interlobar fissures on chest radiograph, with a rapid improvement after fiberoptic bronchoscopy and/or chest physiotherapy and/or mechanical ventilation.

- COPD decompensation was defined as a life-threating exacerbation of COPD; exacerbation was defined by the presence of at least 2 of the following signs and symptoms: change in baseline dyspnea, cough, and sputum quantity or purulence. 
TABLE 1. Patient characteristics

\begin{tabular}{|c|c|c|c|c|}
\hline Characteristic & All patients $(\mathrm{N}=\mathbf{5 4 3})$ & $\begin{array}{c}\text { Intubated } \\
\text { patients }(n=89 ; 16 \%)\end{array}$ & $\begin{array}{c}\text { Non-reintubated } \\
\text { patients }(n=454 ; 84 \%)\end{array}$ & $P$ value \\
\hline Male sex, n $(\%)$ & $398(73)$ & $72(82)$ & $326(72)$ & .08 \\
\hline Median age [Q1-Q3], y & $63[56-69]$ & $65[57-70]$ & $62[56-69]$ & .13 \\
\hline Smoker, n $(\%)$ & $468(86)$ & $74(83)$ & $394(87)$ & .36 \\
\hline Active smoker, $\mathrm{n}(\%)$ & $116(21)$ & $24(27)$ & $92(20)$ & .16 \\
\hline Obesity, n (\%) & $52(10)$ & $12(15)$ & $40(10)$ & .18 \\
\hline Chronic alcohol abuse, $\mathrm{n}(\%)$ & $89(16)$ & $21(24)$ & $68(15)$ & .04 \\
\hline Diabetes mellitus, $\mathrm{n}(\%)$ & $52(10)$ & $15(17)$ & $37(8)$ & .01 \\
\hline Systemic arterial hypertension, $\mathrm{n}(\%)$ & $167(31)$ & $36(41)$ & $131(29)$ & .03 \\
\hline Chronic cardiac disease, $\mathrm{n}(\%)$ & $96(18)$ & $29(33)$ & $67(15)$ & $<.01$ \\
\hline COPD, n $(\%)$ & $256(47)$ & $50(56)$ & $206(45)$ & .06 \\
\hline Median CCI [Q1-Q3] & $5[4-6]$ & $6[5-7]$ & 5 [4-6] & $<.01$ \\
\hline Median preoperative FEV1 [Q1-Q3], \% & 77 [69-92] & $75[62-85]$ & $78[69-93]$ & $<.01$ \\
\hline Median FEV1/FVC [Q1-Q3], \% & 72 [64-79] & $73[65-80]$ & $72[64-78]$ & .36 \\
\hline Median ppoFEV1 [Q1-Q3], \% & $49[42-57]$ & $46[39-52]$ & $50[43-57]$ & $<.01$ \\
\hline Median lung DLCO/VA [Q1-Q3] $(\mathrm{n}=366), \%$ & $79[68-91]$ & $77[55-91]$ & 79 [69-91] & .38 \\
\hline History of previous lung cancer, $\mathrm{n}(\%)$ & $35(6)$ & $9(10)$ & $26(6)$ & .12 \\
\hline History of other cancers, $\mathrm{n}(\%)$ & $70(13)$ & $17(19)$ & $53(12)$ & .06 \\
\hline Right-side pneumonectomy, n (\%) & $244(45)$ & $58(65)$ & $186(41)$ & $<.01$ \\
\hline Pathology & & & & $<.01$ \\
\hline $\mathrm{SCC}, \mathrm{n}(\%)$ & $243(45)$ & $43(48)$ & $200(44)$ & \\
\hline ADK & $153(28)$ & $17(19)$ & $136(30)$ & \\
\hline Other histotypes & $116(21)$ & $17(20)$ & $99(22)$ & \\
\hline Mesothelioma & $24(4)$ & $7(8)$ & $17(4)$ & \\
\hline Metastasis & $4(0.7)$ & $2(2)$ & $2(0.4)$ & \\
\hline Benign disease & $3(0.5)$ & $3(3.4)$ & $0(0)$ & \\
\hline Neoadjuvant chemotherapy, $\mathrm{n}(\%)$ & $181(33)$ & $26(29)$ & $155(34)$ & .37 \\
\hline Neoadjuvant radiotherapy, n (\%) & $12(2)$ & $2(2)$ & $10(2)$ & 1 \\
\hline ASA score III to IV $(n=421), n(\%)$ & $165(39)$ & $57(64)$ & $108(24)$ & $<.01$ \\
\hline Extended pneumonectomy, n (\%) & $313(58)$ & $56(63)$ & $198(44)$ & .71 \\
\hline Extrapleural pneumonectomy, n (\%) & $37(7)$ & $15(17)$ & $22(5)$ & $<.01$ \\
\hline Completion pneumonectomy, $\mathrm{n}(\%)$ & $36(7)$ & $10(11)$ & $26(72)$ & .06 \\
\hline Carinal resection, $\mathrm{n}(\%)$ & $32(6)$ & $12(14)$ & $20(4)$ & $<.01$ \\
\hline$p$ stage $(\mathrm{n}=499), \mathrm{n}(\%)$ & & 77 & 422 & .34 \\
\hline IA & $23(5)$ & $5(7)$ & $18(4)$ & \\
\hline IB & $25(5)$ & $6(8)$ & $19(4)$ & \\
\hline IIA & $56(11)$ & $4(5)$ & $52(12)$ & \\
\hline IIB & $67(13)$ & 15 (19) & $52(12)$ & \\
\hline IIIA & $271(54)$ & $34(44)$ & $237(56)$ & \\
\hline IIIB & $36(7)$ & $9(12)$ & $27(6)$ & \\
\hline IV & $21(4)$ & $4(5)$ & $17(4)$ & \\
\hline
\end{tabular}

Continuous variables are reported as median [interquartile range, Q1-Q3]. Categorial variables are reported as n (\%). Q1-Q3, Interquartile range, quartile 1 to $3 ; C O P D$, chronic obstructive pulmonary disease; $C C I$, Charlson comorbidity index; $F E V I$, forced expiratory volume in 1 second; $F V C$, forced vital capacity; ppoFEVI, predictive postoperative forced expiratory volume in 1 second; $D L C O$, diffusing capacity for carbon monoxide; $V A$, alveolar volume; SCC, squamous-cell carcinoma; $A D K$, adenocarcinoma; $A S A$, American Society of Anesthesiologists; $p$ stage, pathologic stage. 
TABLE 2. Multivariate analysis to assess risk of reintubation: Preoperative and operative factors independently associated with postoperative reintubation $(n=425)$

\begin{tabular}{llc}
\hline \multicolumn{1}{c}{ Characteristic } & OR $(\mathbf{9 5} \%$ CI $)$ & $\boldsymbol{P}$ value \\
\hline Sex (women vs men) & $1.25(0.61-2.55)$ & .54 \\
Side (right vs left) & $2.29(1.24-4.22)$ & $<.01$ \\
Extrapleural pneumonectomy & $8.36(3.31-21.11)$ & $<.01$ \\
ppoFEV1 & $0.99(0.96-1.01)$ & .35 \\
Carinal resection & $3.23(1.26-8.29)$ & .01 \\
CCI & $1.35(1.14-1.61)$ & $<.01$ \\
Chronic alcohol abuse & $1.12(0.54-2.31)$ & .75 \\
Diabetes & $1.17(0.49-2.80)$ & .72 \\
Systemic hypertension & $1.19(0.64-2.20)$ & .58 \\
Chronic cardiac disease & $2.15(1.08-4.25)$ & .03 \\
\hline
\end{tabular}

$O R$, Odds ratio; $C I$, confidence interval; ppoFEVI, predictive postoperative forced expiratory volume in 1 second; $\mathrm{CCI}$, Charlson comorbidity index.

- Cardiac pulmonary edema was defined by an increased capillary hydrostatic pressure secondary to elevated pulmonary venous pressure, in turn secondary to cardiac dysfunction.

- Hemorrhagic shock was defined by excessive bleeding associated with arterial hypotension not responding to fluid infusion and resulting in the use of vasopressors.

- Sepsis was defined by a systemic inflammatory response of the host to an infectious aggression.

- AKI was defined by the "risk stage" of the Risk, Injury, Failure, Loss of kidney function, End-stage kidney disease (RIFLE) classification: increase of creatinine level by 1.5 compared with its concentration before surgery.

- Acute coronary syndrome was defined by an ischemic cardiac dysfunction due to coronary stenosis or emboli.

\section{RESULTS}

Five hundred forty-three patients underwent pneumonectomy in the time frame of the study. Baseline characteristics of the whole population are detailed in Table 1. Eighty-nine patients $(16 \%)$ needed reintubation and IMV within
30 postoperative days. In 53 of 89 patients who required reintubation $(60 \%)$, ARF was initially managed with noninvasive ventilation and intubation was necessary because of failure of NIV. Sixty of 89 reintubated patients (67\% of IMV patients and $11 \%$ of all pneumonectomy patients) developed ARDS. Mild, moderate, and severe ARDS occurred in $3(5 \%), 21(35 \%)$, and $36(60 \%)$ patients, respectively.

\section{Risk Factors for Reintubation}

Comparison of baseline characteristics between patients requiring $(\mathrm{n}=89,16 \%)$ or not $(\mathrm{n}=454,84 \%)$ reintubation is shown in Table 1. Patients requiring reintubation had a more frequent history of alcohol abuse $(P=.04)$, diabetes $(P=.01)$, hypertension $(P=.03)$, chronic cardiac disease $(P<.01)$. CCI was thus higher in reintubated patients $(P<.01)$. Reintubated patients had lower FEV1 $(P<.01)$ and lower predictive ppoFEV1 $(P<.01)$, more frequent right-side disease $(P<.01)$, and had more frequently undergone extrapleural pneumonectomy $(P<.01)$ or carinal resection $(P<.01)$.

A multivariate analysis model was built taking into account ppoFEV1 but not FEV1 (because of the strong interaction between the 2 parameters; Table 2). Right-side pneumonectomy (odds ratio [OR], 2.29; 95\% confidence interval $[\mathrm{CI}], 1.24-4.22)$, extrapleural pneumonectomy (OR, 8.36; 95\% CI, 3.31-21.11), carinal resection (OR, 3.23; 95\% CI, 1.26-8.29), CCI (OR, $1.35 ; 95 \% \mathrm{CI}$, 1.14-1.61), and chronic cardiac disease (OR, 2.15; 95\% CI, 1.08-4.25) were identified as independent risk factors. Results were consistent in a sensitivity analysis involving stepwise selection (data not shown).

\section{Outcomes}

Postoperative complications in the whole population and in the groups of reintubated versus nonreintubated patients

TABLE 3. Postoperative complications associated or not with reintubation

\begin{tabular}{|c|c|c|c|c|}
\hline & $\begin{array}{l}\text { All patients } \\
(N=543)\end{array}$ & $\begin{array}{l}\text { Intubated patients } \\
\quad(\mathbf{n}=89 ; 16 \%)\end{array}$ & $\begin{array}{l}\text { Non-reintubated patients } \\
\quad(\mathrm{n}=\mathbf{4 5 4} ; \mathbf{8 4} \%)\end{array}$ & $P$ value \\
\hline \multicolumn{5}{|l|}{ Indications for ventilation } \\
\hline \multicolumn{5}{|l|}{ Acute respiratory failure } \\
\hline Pneumonia & $98(18)$ & $57(64)$ & $41(9)$ & $<.01$ \\
\hline Atelectasis & $32(6)$ & $13(15)$ & $19(4)$ & $<.01$ \\
\hline COPD decompensation & $40(7)$ & $5(7)$ & $35(8)$ & .49 \\
\hline Cardiac pulmonary edema & $28(5)$ & $9(10)$ & $19(4)$ & .04 \\
\hline Pulmonary embolism & $3(0.6)$ & $2(2)$ & $1(0.2)$ & .07 \\
\hline Hemorrhagic shock & $23(4)$ & $16(18)$ & $7(2)$ & $<.01$ \\
\hline \multicolumn{5}{|l|}{ Nonrespiratory complications } \\
\hline Sepsis & $67(12)$ & $63(71)$ & $4(1)$ & $<.01$ \\
\hline Acute kidney injury & $48(9)$ & $33(37)$ & $15(3)$ & $<.01$ \\
\hline Acute coronary syndrome & $12(2)$ & $12(14)$ & $0(0)$ & $<.01$ \\
\hline NIV for ARF & $94(17)$ & $53(60)$ & $41(9)$ & $<.01$ \\
\hline
\end{tabular}

Data are reported as n (\%). COPD, Chronic obstructive pulmonary disease; $N I V$, noninvasive ventilation; $A R F$, acute respiratory failure. 
TABLE 4. Baseline characteristics of the survivors versus nonsurvivors among the 89 reintubated patients

\begin{tabular}{|c|c|c|c|c|}
\hline & All patients $(N=89)$ & $\begin{array}{l}\text { Survivors at day } 30 \\
\quad(n=55 ; 62 \%)\end{array}$ & $\begin{array}{c}\text { Nonsurvivors at day } 30 \\
(n=34 ; 38 \%)\end{array}$ & $P$ value \\
\hline Male sex, n (\%) & $72(80)$ & $45(81)$ & $27(79)$ & .78 \\
\hline Median age [Q1-Q3], y & $65[57.5-71]$ & $65[57.5-71]$ & $65[57-70]$ & $<.01$ \\
\hline Smoker, n (\%) & $73(83)$ & $52(87)$ & $22(76)$ & .99 \\
\hline Active smoker, $\mathrm{n}(\%)$ & $24(28)$ & $17(30)$ & $8(24)$ & .61 \\
\hline Median BMI [Q1-Q3] & $24.6[22-27]$ & $25[20.1-27.5]$ & $24.5[23-27.6]$ & .81 \\
\hline Chronic alcohol abuse, n (\%) & $21(23)$ & $15(27)$ & $5(15)$ & .26 \\
\hline Diabetes mellitus, $\mathrm{n}(\%)$ & $15(18)$ & $12(22)$ & $4(12)$ & .36 \\
\hline Chronic cardiac disease, $\mathrm{n}(\%)$ & $29(34)$ & $16(29)$ & $14(41)$ & .35 \\
\hline Previous nonpulmonary cancer, $\mathrm{n}(\%)$ & $17(19)$ & $7(13)$ & $10(29)$ & .06 \\
\hline Median CCI [Q1-Q3] & $6[5-7]$ & 6 [4-7] & 6 [5-7] & .24 \\
\hline Previous lung cancer, n (\%) & $9(9)$ & $4(7)$ & $4(12)$ & .73 \\
\hline COPD, n $(\%)$ & $50(57)$ & $28(50)$ & $23(68)$ & .18 \\
\hline Median preoperative FEV1 [Q1-Q3], \% & $75[62-85]$ & 73 [57-85] & $77[66-85]$ & .27 \\
\hline Median FEV1/FVC [Q1-Q3], \% & $73[65-80]$ & $75[68-81]$ & $71[65-78]$ & .38 \\
\hline Median ppoFEV1 [Q1-Q3], \% & 46 [39-52] & 45 [39-52] & 45 [39-47] & .36 \\
\hline Right-side pneumonectomy, n (\%) & $58(65)$ & $31(35)$ & $27(79)$ & .03 \\
\hline Neoadjuvant chemotherapy, n (\%) & $26(29)$ & $15(17)$ & $11(32)$ & .78 \\
\hline Neoadjuvant radiotherapy, $\mathrm{n}(\%)$ & $2(2)$ & $2(4)$ & $0(0)$ & .69 \\
\hline Pneumonectomy, n (\%) & $25(28)$ & $14(26)$ & $11(32)$ & .63 \\
\hline Extended pneumonectomy, n (\%) & $64(72)$ & $41(75)$ & $23(68)$ & .63 \\
\hline ASA score III and IV, n (\%) & $57(64)$ & $34(62)$ & $23(68)$ & .74 \\
\hline
\end{tabular}

Continuous variables are reported as median [interquartile ranges, Q1-Q3]. Categorial variables are reported as n (\%). $Q 1-Q 3$, Interquartile range quartile 1 to $3 ; B M I$, body mass index; $C C I$, Charlson comorbidity index; COPD, chronic obstructive pulmonary disease; $F E V I$, forced expiratory volume in 1 second; $F V C$, forced capacity volume; ppoFEVI, predictive postoperative forced expiratory volume in 1 second; ASA, American Society of Anesthesiologists.

TABLE 5. Characteristics at ICU admission and postoperative complications of survivors versus nonsurvivors among the reintubated patients

\begin{tabular}{|c|c|c|c|c|}
\hline & $\begin{array}{c}\text { All reintubated } \\
\text { patients }(N=89)\end{array}$ & $\begin{array}{c}\text { Survivors at } \\
\text { day } 30(\mathrm{n}=55 ; 62 \%)\end{array}$ & $\begin{array}{c}\text { Nonsurvivors at } \\
\text { day } 30(n=34 ; 38 \%)\end{array}$ & $P$ value \\
\hline Median SAPS2 & $26.5[19-36]$ & 24 [17-32] & 28 [24-39] & .06 \\
\hline Median SOFA at day 1 & $2[1-7]$ & $2[1-7]$ & $2[1-8]$ & .47 \\
\hline Median SOFA at day 3 & 3 [2-9] & $3[1.5-7]$ & $3[2-10]$ & .16 \\
\hline \multicolumn{5}{|l|}{ Indications for reintubation } \\
\hline Acute respiratory failure & $77(87)$ & $45(82)$ & $32(94)$ & .18 \\
\hline Pneumonia & $57(64)$ & $35(64)$ & $22(65)$ & .99 \\
\hline Atelectasis & $13(15)$ & $9(16)$ & $4(12)$ & .77 \\
\hline COPD decompensation & $50(58)$ & $26(47)$ & $24(71)$ & .99 \\
\hline Cardiac pulmonary edema & $9(10)$ & $6(11)$ & $3(9)$ & .99 \\
\hline Pulmonary embolism & $2(2)$ & $2(4)$ & $0(0)$ & .69 \\
\hline Hemorrhagic shock & $16(18)$ & $9(16)$ & $7(21)$ & .82 \\
\hline \multicolumn{5}{|l|}{ Nonrespiratory complications } \\
\hline Sepsis & $63(71)$ & $37(67)$ & $26(77)$ & .49 \\
\hline Acute kidney injury & $33(37)$ & $14(25)$ & $19(56)$ & $<.01$ \\
\hline Acute cerebral stroke & $2(2)$ & $2(4)$ & $0(0)$ & .69 \\
\hline Acute coronary syndrome & $12(14)$ & $5(9)$ & $7(21)$ & .22 \\
\hline ARDS, n (\%) & $60(67)$ & $31(56)$ & $29(85)$ & $<.01$ \\
\hline
\end{tabular}

Continuous variables are reported as median [interquartile ranges, Q1-Q3]. Categorial variables are reported as n (\%). SAPS2, Simplified Acute Physiologic Score 2; SOFA, sepsis-related organ failure assessment; COPD, chronic obstructive pulmonary disease; $A R D S$, acute respiratory distress syndrome. 
TABLE 6. Factors independently associated with 30-day mortality for the 89 reintubated patients

\begin{tabular}{lcc}
\hline \multicolumn{1}{c}{ Factor } & OR $(\mathbf{9 5} \% \mathbf{C I})$ & $\boldsymbol{P}$ value \\
\hline ARDS & $7.33(1.47-36.67)$ & .02 \\
SAPS $2>25$ & $4.57(1.19-17.52)$ & .03 \\
Right-side pneumonectomy & $4.95(1.19-20.54)$ & .03 \\
\hline
\end{tabular}

OR, Odds ratio; $C I$, confidence interval; $A R D S$, acute respiratory distress syndrome; SAPS, simplified acute physiologic score.

are shown in Table 3. In particular, pneumonia $(P<.01)$, atelectasis $(P<.01)$, cardiogenic pulmonary edema $(P=.04)$, hemorrhagic shock $(P<.01)$, sepsis $(P<.01)$, AKI $(P<.01)$, acute coronary syndrome $(P<.01)$, and need for noninvasive ventilation $(P<.01)$ were more frequent in reintubated than in nonreintubated patients.

Thirty-day mortality was $7.7 \%(n=42 / 543)$ in the whole population of pneumonectomy patients, $41.6 \%(n=37 / 89)$ in reintubated patients, and 53.3\% $(\mathrm{n}=32 / 60)$ in ARDS patients. In nonreintubated patients, 30 mortality was $1.1 \%(\mathrm{n}=5 / 454)$. Mortality was significantly higher in patients with severe than in those with mild or moderate ARDS $(63.8 \% ; \mathrm{n}=23 / 36$ vs $25 \% ; \mathrm{n}=6 / 24 ; P<.01)$.

\section{Characteristics and Outcome in the Whole Population of Reintubated Patients}

Comparisons of baseline characteristics between survivors and nonsurvivors among the 89 reintubated patients are shown in Table 4. Nonsurvivors were older $(P<.01)$, had more frequently right pneumonectomy $(P=.03)$. Table 5 shows characteristics at ICU admission, postoperative complications, and the indications for reintubation in survivors and nonsurvivors. AKI and
ARDS were significantly more frequent in nonsurvivors than in survivors (both $P<.01$ ). Logistic regression identified 3 independent predictors of 30-day mortality: ARDS (OR, 7.33; 95\% CI, 1.47-36.67; $P=.02$ ), SAPS2 $>25$ (OR, 4.57; 95\% CI, 1.19-17.52; $P=.03$ ) and right-side pneumonectomy $(\mathrm{OR}, 4.95 ; 95 \% \quad \mathrm{CI}$, 1.19-20.54; $P=.03$; Table 6).

\section{Long-Term Outcome}

Overall survival curves in pneumonectomy patients who required intubation is reported in Figure 1. After surgery, in intubated patients, median survival was 7.7 months $(95 \%$ CI, 1.6-18.7). Five-year survival was $27.1 \%(95 \% \mathrm{CI}$, 17.8-41.4)

\section{DISCUSSION}

In the present study, we provide evidence that early ARF requiring reintubation remains a frequent and severe complication after pneumonectomy, occurring in $16.4 \%$ of cases. ARDS occurred in $11.4 \%$ of patients, with severe ARDS representing more than half of the cases. We identified right-side pneumonectomy, chronic cardiac disease, higher CCI, and specific types of extended pneumonectomy (extrapleural or sleeve) as independent risk factors for reintubation. Our results suggest that neither FEV1 nor predicted postoperative FEV1 were associated with need for reintubation.

Early mortality of patients with ARF requiring IMV was $41.6 \%$ and it was $53.3 \%$ when patients developed ARDS. Only 3 factors were independently associated with early mortality: right pneumonectomy, SAPS2 at ICU admission, and occurrence of ARDS.

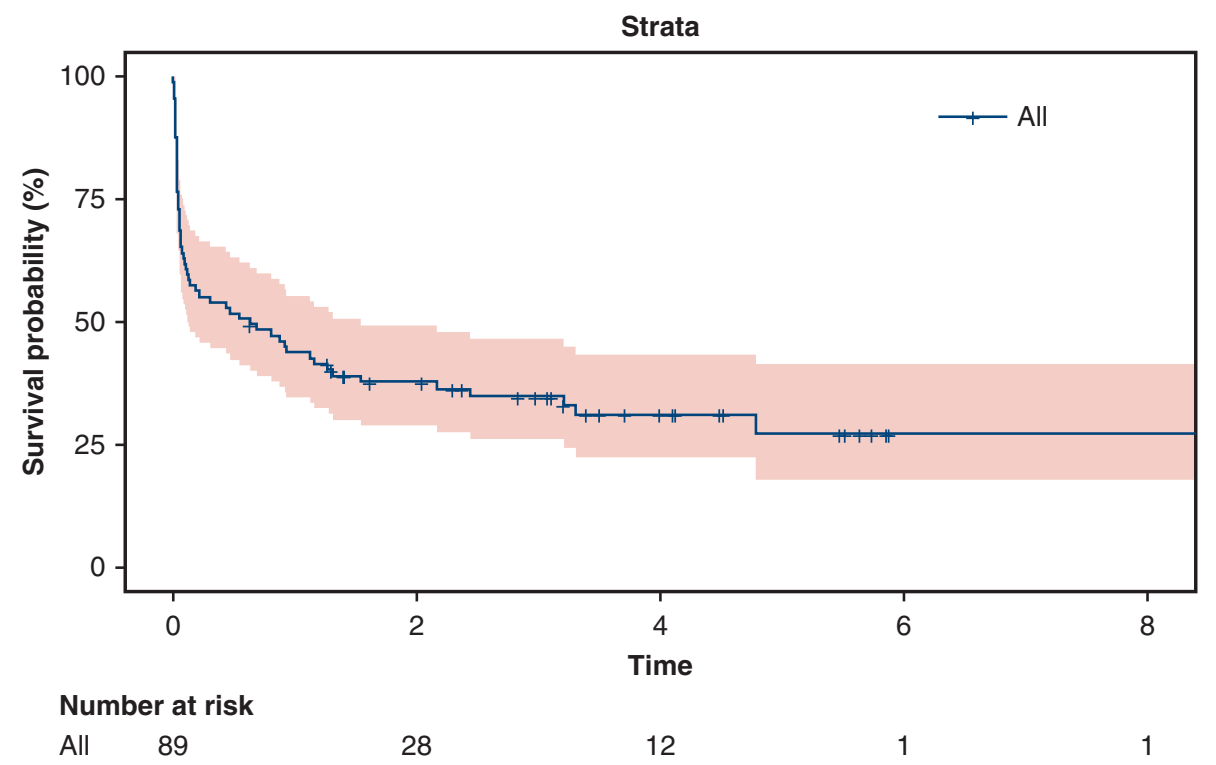

FIGURE 1. Overall survival. Kaplan-Meier curve in pneumonectomy patients requiring intubation. Intubated patients, $\mathrm{n}=89$; median survival, 7.7 months; $95 \%$ confidence interval, 1.6-18.7; 5-year survival, $27.1 \%$; 95\% confidence interval, 17.8-41.4. 
Thus, although our prevalence of ARF is comparable with rates previously reported in smaller series, ${ }^{8,9,24}$ our mortality figure in reintubated patients was higher than previously reported: for example, Hamaji and colleagues $^{19}$ reported a 30 -day operative mortality of $26 \%$. As already reported by our team, ${ }^{25}$ NIV is very efficient in hypercapnic postoperative ARF (after all types of lung resection), avoiding the need for intubation in a large percentage of cases. ${ }^{26}$ Of 89 patients who required reintubation, $60 \%$ were initially managed with NIV and IMV might be considered as failure of NIV. Most of our intubated patients had hypoxemic ARF, a situation with a poorer prognosis than hypercapnic ARF. ${ }^{25}$ Regarding NIV use before reintubation, no difference was found between survivors and nonsurvivors.

Our study recognized only a few factors significantly associated with early mortality. Age and SAPS2 score at admission in the ICU were associated with early mortality, as reported in many other studies. ${ }^{13,23,27}$ Right-side pneumonectomy was significantly associated with a poor outcome. Several studies reported a higher postoperative mortality for the right side, ${ }^{13,28,29}$ which could be related to several factors, in particular the usual predominance of the right lung in terms of perfusion and ventilation and the higher postoperative pulmonary arterial pressure after right than left pneumonectomy. ${ }^{30}$ Almost all our patients had a preoperative lung perfusion scan and a transthoracic echocardiography. These recommended noninvasive tests might not be sensitive enough to predict postpneumonectomy pulmonary hypertension, which has been reported to be associated with postpneumonectomy ARDS. ${ }^{31}$

We observed, in our reintubated patients, a 5-year survival rate of $27.1 \%$, confirming the poor outcome of these patients. However, this result compares favorably with previously reported mortality rates for medical cancer patients with ARF requiring mechanical ventilation. ${ }^{31}$ Of note, a recently published study reported a 5-year survival rate after pneumonectomy for lung cancer of approximately $35 \% .^{13}$

\section{Strengths and Limitations of the Study}

We were able to collect data on many baseline characteristics, respiratory function, and pathologic parameters, but also intraoperative and postoperative care data. To our knowledge, this is the first study to consider all of these parameters to analyze outcome of patients with postpneumonectomy ARF.

The main limitation is represented by the retrospective character of the study and the existence of some missing data. However, despite the retrospective character of the study, all of the patients operated on in the studied period could be included. Furthermore, there was no missing data on main outcome parameters. Missing data concerned mainly some functional parameters: for example DLCO was not measured systematically in all of the patients and was thus available in $67 \%$ of cases, whereas perfusion lung scan was performed in $78 \%$ of the patients. Another limitation could be represented by the relatively long time frame of the study period (10 years): although the study was monocentric, we cannot exclude that some evolution in patient management occurred between the beginning and the end of the period.

\section{CONCLUSIONS}

Our study highlights that management of ARF in pneumonectomy patients remains challenging. Right-side pneumonectomy, chronic cardiac disease, higher CCI, and specific types of extended pneumonectomy (extrapleural or sleeve) were identified as independent risk factors for reintubation, whereas neither FEV1 nor predicted postoperative FEV1 were significantly associated with need of reintubation. These elements could help identify high-risk patients for better selection and adequate counseling of patients and families. Studies aiming at identifying other patient-related factors (eg, morphometric assessment, including BMI, ${ }^{34}$ total psoas area, ${ }^{34}$ normalized pulmonary artery diameter ${ }^{35}$ ), possibly influencing postpneumonectomy immediate outcome are needed. Combining such parameters with those reported in the present study in a large cohort of patients could allow developing a specific risk score for postpneumonectomy ARF and ARDS occurrence.

\section{Conflict of Interest Statement}

Authors have nothing to disclose with regard to commercial support.

\section{References}

1. Schussler O, Alifano M, Dermine H, Strano S, Casetta A, Sepulveda S, et al Postoperative pneumonia after major lung resection. Am J Respir Crit Care Med. 2006;173:1161-9.

2. Algar FJ, Alvarez A, Salvatierra A, Baamonde C, Aranda JL, López-Pujol FJ, et al. Predicting pulmonary complications after pneumonectomy for lung cancer. Eur J Cardiothorac Surg. 2003;23:201-8.

3. Bernard A, Deschamps C, Allen MS, Miller DL, Trastek VF, Jenkins GD, et al. Pneumonectomy for malignant disease: factors affecting early morbidity and mortality. J Thorac Cardiovasc Surg. 2001;121:1076-82.

4. Duque JL, Ramos G, Castrodeza J, Cerezal J, Castanedo M, Yuste MG, et al. Early complications in surgical treatment of lung cancer: a prospective, multicenter study. Grupo Cooperativo de Carcinoma Broncogénico de la Sociedad Española de Neumología y Cirugía Torácica. Ann Thorac Surg. 1997;63:944-50.

5. Stéphan F, Boucheseiche S, Hollande J, Flahault A, Cheffi A, Bazelly B, et al. Pulmonary complications following lung resection: a comprehensive analysis of incidence and possible risk factors. Chest. 2000;118:1263-70.

6. Strand TE, Rostad H, Damhuis RA, Norstein J. Risk factors for 30-day mortality after resection of lung cancer and prediction of their magnitude. Thorax. 2007; 62:991-7.

7. Rosen JE, Hancock JG, Kim AW, Detterbeck FC, Boffa DJ. Predictors of mortality after surgical management of lung cancer in the National Cancer Database. Ann Thorac Surg. 2014;98:1953-60.

8. Dulu A, Pastores SM, Park B, Riedel E, Rusch V, Halpern NA. Prevalence and mortality of acute lung injury and ARDS after lung resection. Chest. 2006; 130:73-8. 
9. Tang SS, Redmond K, Griffiths M, Ladas G, Goldstraw P, Dusmet M. The mortality from acute respiratory distress syndrome after pulmonary resection is reducing: a 10-year single institutional experience. Eur J Cardiothorac Surg. 2008;34:898-902.

10. Kutlu CA, Williams EA, Evans TW, Pastorino U, Goldstraw P. Acute lung injury and acute respiratory distress syndrome after pulmonary resection. Ann Thorac Surg. 2000;69:376-80.

11. Ruffini E, Parola A, Papalia E, Filosso PL, Mancuso M, Oliaro A, et al. Frequency and mortality of acute lung injury and acute respiratory distress syndrome after pulmonary resection for bronchogenic carcinoma. Eur J Cardiothorac Surg. 2001;20:30-6.

12. Toufektzian L, Patris V, Sepsas E, Konstantinou M. Does postoperative mechanical ventilation predispose to bronchopleural fistula formation in patients undergoing pneumonectomy? Interact Cardiovasc Thorac Surg. 2015;21:379-82.

13. Janet-Vendroux A, Loi M, Bobbio A, Lococo F, Lupo A, Ledinot P, et al. Which is the role of pneumonectomy in the era of parenchymal-sparing procedures? Early/long-term survival and functional results of a single-center experience. Lung. 2015;193:965-73.

14. Alam N, Park BJ, Wilton A, Seshan VE, Bains MS, Downey RJ, et al. Incidence and risk factors for lung injury after lung cancer resection. Ann Thorac Surg. 2007;84:1085-91.

15. Jeon K, Yoon JW, Suh GY, Kim J, Kim K, Yang M, et al. Risk factors for postpneumonectomy acute lung injury/acute respiratory distress syndrome in primary lung cancer patients. Anaesth Intensive Care. 2009;37:14-9.

16. Kim JB, Lee SW, Park SI, Kim YH, Kim DK. Risk factor analysis for postoperative acute respiratory distress syndrome and early mortality after pneumonectomy: the predictive value of preoperative lung perfusion distribution. J Thorac Cardiovasc Surg. 2010;140:26-31.

17. Licker M, de Perrot M, Spiliopoulos A, Robert J, Diaper J, Chevalley C, et al. Risk factors for acute lung injury after thoracic surgery for lung cancer. Anesth Analg. 2003;97:1558-65.

18. Parquin F, Marchal M, Mehiri S, Hervé P, Lescot B. Post-pneumonectomy pulmonary edema: analysis and risk factors. Eur J Cardiothorac Surg. 1996;10: 929-32.

19. Hamaji M, Keegan MT, Cassivi SD, Shen KR, Wigle DA, Allen MS, et al. Outcomes in patients requiring mechanical ventilation following pneumonectomy. Eur J Cardiothorac Surg. 2014;46:4-19.

20. Institut National Du Cancer. Cancers bronchopulmonaires et pleuraux. Recommandations et outils d'aide à la pratique. Available at: http://www.e-cancer.fr/ Professionnels-de-sante/Recommandations-et-outils-d-aide-a-la-pratique/Cancersbronchopulmonaires-et-pleuraux. Accessed March 15, 2016.

21. Le Gall JR, Lemeshow S, Saulnier F. A new Simplified Acute Physiology Score (SAPS II) based on a European/North American multicenter study. JAMA. 1993; 270:2957-63.

22. Vincent JL, Moreno R, Takala J, Willatts S, De Mendonça A, Bruining H, et al. The SOFA (Sepsis-related Organ Failure Assessment) score to describe organ dysfunction/failure. On behalf of the Working Group on Sepsis-Related Prob- lems of the European Society of Intensive Care Medicine. Intensive Care Med. 1996;22:707-10.

23. ARDS Definition Task Force, Ranieri VM, Rubenfeld GD, Thompson BT, Ferguson ND, Caldwell E, Fan E, et al. Acute respiratory distress syndrome: the Berlin Definition. JAMA. 2012;307:2526-33.

24. Licker M, Diaper J, Villiger Y, Spiliopoulos A, Licker V, Robert J, et al. Impact of intraoperative lung-protective interventions in patients undergoing lung cancer surgery. Crit Care. 2009;13:R41.

25. Lefebvre A, Lorut C, Alifano M, Dermine H, Roche N, Gauzit R, et al. Noninvasive ventilation for acute respiratory failure after lung resection: an observational study. Intensive Care Med. 2009;35:663-70.

26. Auriant I, Jallot A, Hervé P, Cerrina J, Le Roy Ladurie F, Fournier JL, et al. Noninvasive ventilation reduces mortality in acute respiratory failure following lung resection. Am J Respir Crit Care Med. 2001;164:1231-5.

27. Pricopi C, Mordant P, Rivera C, Arame A, Foucault C, Dujon A, et al. Postoperative morbidity and mortality after pneumonectomy: a 30-year experience of 2064 consecutive patients. Interact Cardiovasc Thorac Surg. 2015;20:316-32.

28. Darling GE, Abdurahman A, Yi QL, Johnston M, Waddell TK, Keshavjee PA, et al. Risk of a right pneumonectomy: role of bronchopleural fistula. Ann Thorac Surg. 2005;79:433-7.

29. Thomas PA, Berbis J, Baste JM, Le Pimpec-Barthes F, Tronc F, Falcoz PE, et al. Pneumonectomy for lung cancer: contemporary national early morbidity and mortality outcomes. J Thorac Cardiovasc Surg. 2015;149:73-82.

30. Foroulis CN, Kotoulas CS, Kakouros S, Evangelatos G, Chassapis C, Konstantinou M, et al. Study on the late effect of pneumonectomy on right heart pressures using Doppler echocardiography. Eur J Cardiothorac Surg. 2004;26:508-14.

31. van der Werff YD, van der Houwen HK, Heijmans PJ, Duurkens VA, Leusink HA, van Heesewijk HP, et al. Postpneumonectomy pulmonary edema. A retrospective analysis of incidence and possible risk factors. Chest. 1997; 111:1278-84.

32. Bigatello LM, Allain R, Gaissert HA. Acute lung injury after pulmonary resection. Minerva Anestesiol. 2004;70:159-66.

33. Lewis JW, Bastanfar M, Gabriel F, Mascha E. Right heart function and prediction of respiratory morbidity in patients undergoing pneumonectomy with moderately severe cardiopulmonary dysfunction. J Thorac Cardiovasc Surg. 1994;108:169-75.

34. Hervochon R, Bobbio A, Guinet C, Mansuet-Lupo A, Rabbat A, Régnard JF, et al. Body mass index and total psoas area affect outcomes in patients undergoing pneumonectomy for cancer. Ann Thorac Surg. 2017;103:287-95.

35. Peretti M, Hervochon R, Loi M, Blanc K, Roche N, Alifano M. Predictors of post-pneumonectomy respiratory failure and ARDS: usefulness of normalized pulmonary artery diameter. Intensive Care Med. May 11, 2018 [Epub ahead of print].

Key Words: pneumonectomy, acute respiratory failure, outcome, lung cancer 